\title{
Metabolic response to dietary supplementation with iron and folic acid in the rat
}

\section{Abstract}

Folic acid (FA) and iron absorption is performed by transporters encoded by the Slc46al and Slc11a2 genes, respectively. The aim of this study was to determine how FA and iron deficiency in rats, and subsequent supplementation of the animals' diet with these nutrients, affects Slc11a2 and Slc46a1 gene expression and the metabolic biomarkers of FA and iron status.

150 female Wistar rats were assigned to a group fed a diet deficient in iron and FA $(\mathrm{D}, \mathrm{n}=120)$ or to a group fed a control diet $(\mathrm{C}$, $\mathrm{n}=30$, AIN-93M) for 28 days. After this period, the rats were randomized to a group fed for 2, 10, or 21 days with a diet deficient in FA and supplemented with iron (DFE), a diet deficient in iron and supplemented with FA (DFOL), a diet supplemented with iron and FA (FEFOL), or a diet deficient in iron and folic (D).

Transcript and protein levels were determined in the duodenum using the real-time PCR and western blot methods, respectively. FA concentrations in serum were assessed using the electrochemiluminescence method, and homocysteine concentrations were determined with an enzymatic assay. Iron metabolism was evaluated by measuring the total and unsaturated iron-binding capacity (TIBC and UIBC) in serum, and iron concentrations were determined using a photometric method. Hepcidin concentrations were measured in plasma with an immunoenzymatic method. Morphological parameters of the blood were determined with a Sysmex XT-4000 analyzer.

The D group had higher Slc11a2 transcript levels than the DFE group at every time-point $(\mathrm{p}<0.01)$. There were higher Slc46a1 mRNA levels in the DFE group than in the FEFOL group at the third time-point $(p<0.05)$. We did not observe any differences in PCFT or DMT1 protein abundance. The DFOL, FEFOL, and C groups had higher serum FA concentrations at the second and third time-points $(p<0.001)$, as well as the lowest homocysteine concentrations $(p<0.001)$. TIBC concentrations were lowest in the DFE and the FEFOL groups at the final time-point $(\mathrm{p}<0.001)$. We did not observe any differences in hepcidin concentrations. Simultaneous supplementation with FA and iron resulted in significantly higher hemoglobin concentrations at the third time-point $(\mathrm{p}<0.01)$.

This study shows that dietary FA and iron deficiency, and subsequent supplementation with moderate amounts of these nutrients, may affect the transcription but not the protein abundance of FA and iron transporters in the rat duodenum.

Funding: National Science Center, Poland (2015/17/B/NZ7/02952).

\section{Conflict of Interest}

There is no conflict of interest 